\title{
A MICROSCOPIC DUAL-REGIME MODEL FOR SINGLE-LANE ROUNDABOUTS
}

\author{
Estelle Chevallier, Ludovic Leclercq (chevallier@entpe.fr) \\ Laboratoire d'Ingénierie Circulation Transport \\ LICIT - ENTPE / INRETS - Université de Lyon \\ Rue Maurice Audin, 69518 Vaulx-en-Velin Cedex - France \\ Tel: +33472047239 / Fax: +33472047712
}

\begin{abstract}
Most of microsimulation tools used to model roundabouts encompass classical gapacceptance algorithms to represent the insertion of approaching vehicles into the circulatory roadway. However, these algorithms fail to reproduce the average priority sharing process experimentally observed when the circulatory roadway is congested. This paper fills this shortage by proposing an integrated microscopic framework with: (i) a gap-acceptance algorithm giving relevant capacity estimates in uncongested regime; (ii) a probabilistic ratebased insertion decision module in congested regime. In this framework the car-following model can be implemented independently of the insertion decision making process. Moreover, its direct influence on the insertion decision model is released in congested regime thanks to a relaxation procedure. The obtained simulation results are convincing compared to on-field data collected at different sites for both peak and off-peak periods.
\end{abstract}

\section{INTRODUCTION}

Microsimulation models provide flexible and user-definable features to assess traffic operations of roundabouts. Available commercial simulation packages listed by the Federal Highway Administration (Robinson, 2000) such as VISSIM, CORSIM, INTEGRATION, 
PARAMICS or SIMTRAFFIC, represent roundabouts as a series of yield-controlled Tintersections. Two key elements can be customized to reflect roundabouts' characteristics: (i) the car-following algorithm which simulates vehicle trajectories on the circulatory roadway; (ii) the gap-acceptance algorithm which models the yield-on-entry operation.

Lots of studies have been conducted to compare the results given by microsimulation tools with analytical models like aaSIDRA (Akçelik, 2005) or RODEL (NYSDOT, 2002). It is often concluded that microsimulation models are well-suited for capturing the effects of nearby intersections (Bared and Edara, 2005; Trueblood and Dale, 2003) or for modelling complex vehicle interactions at high-capacity multilane roundabouts (Stanek and Milam, 2005). However, concerns are often expressed regarding the sensitivity of the simulated results to parameter values (Akçelik and Besley, 2001). Moreover, the calibration task to either on-field data or analytical models can be cumbersome (Smith et al., 2007). This usually induces large variations in capacity and delay estimates produced by different simulation models as reported by Tian et al. (2006).

The calibration issues and profusion of model parameters are not the only drawbacks of microsimulation tools. Criticisms about their ability to model merging behaviours when traffic conditions downstream of the conflict point are congested (congested regime) have been formulated (Hidas, 2005b). Chevallier and Leclercq (2008) have recently stressed two possible error factors: (i) the numerical viscosity appearing in the car-following algorithm which induces errors in the insertion decision output; (ii) the constraints imposed by the carfollowing algorithm on the gap-acceptance process. Consequently, the ratio of the simulated entry flow over the simulated circulating flow is dependent on both the simulation time-step and the downstream capacity. Particularly, it tends towards zero when the downstream 
congestion becomes too severe. These results are inconsistent with on-field observations. Indeed, Cassidy and Ahn (2005) have revealed that queued vehicles from both links enter the merge in some fixed sharing ratio in congestion, $\gamma$, independent of the severity of the congestion. These findings match Troutbeck's observations at congested urban roundabouts (Troutbeck, 2002). It appears that approaching vehicles do not respect the give-way rule anymore. They rather adopt a deterministic behaviour due to: (i) a reduction in the risk associated with the insertion manoeuvre because of lower speeds on the circulatory roadway; (ii) more uniform headways because of queues on the roundabout. As a result, merging behaviours in congested regime completely differ from the classical gap-acceptance process which occurs when traffic on the circulatory roadway is in free-flow condition (uncongested regime). Hence, for being consistent, a roundabout simulation model should encompass two distinct merging algorithms for each regime.

The goal of this paper is to propose a parsimonious microscopic model for single-lane roundabouts able to capture both uncongested and congested regimes. It is based on: (i) a classical and easy-to-calibrate gap-acceptance algorithm in uncongested regime; (ii) a ratebased probabilistic insertion decision module in congested regime.

The first part of this paper will outline the key components of the proposed framework. Then, the model will be validated in terms of flows and average delays against experimental data.

\section{PROPOSED MICROSCOPIC FRAMEWORK}

\subsection{Overview of the framework}

A $k$-leg roundabout is split into a series of merging areas. The $i^{\text {th }}$ merging area $(i \in[1, k])$ is composed of an approach link $\left(A_{i}\right)$, an upstream circulatory link $\left(I_{i}\right)$, a downstream 
circulatory link $\left(C_{i}\right)$ and, potentially, a departure link $\left(D_{i}\right)$. This is illustrated in figure 1 . In the sequel, we define:

- the first approaching vehicle on link $A_{i}$ as the entering vehicle, $e$;

- the first vehicle on either link $C_{i-1}$ or link $I_{i}$ as the follower, $f$;

- the last vehicle on link $C_{i}$, as the leader, $l$.

The length of any link $R_{i},(R \in\{A, D, C, I\})$ is noted $L_{i}^{R}$.
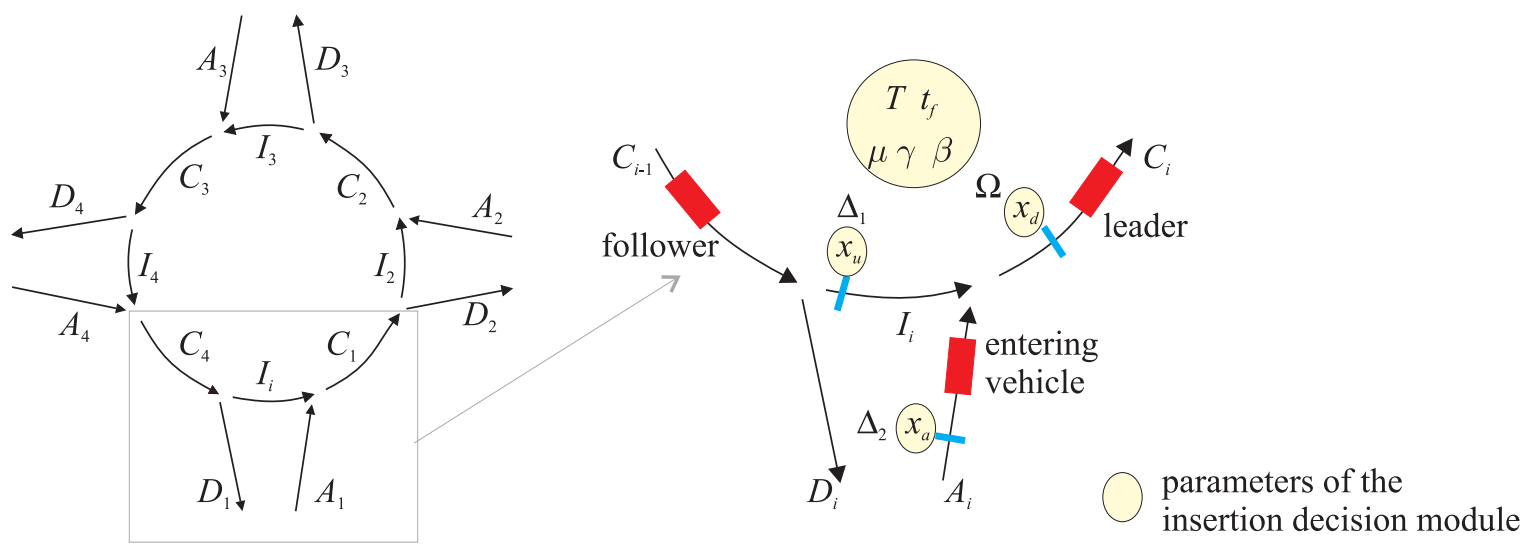

Fig.1. Roundabout modelling

Traffic sates on the roundabout are described by a triangular fundamental diagram which requires only three parameters: (i) the free-flow speed $u$; (ii) the minimum spacing in congestion $s_{0}$ (the inverse of the jam density) and (iii) the wave speed in congestion $w$. Note that these three parameters can vary for each link; we will add a superscript $A, D, C$ or $I$ to lift the ambiguity. Moreover, the maximum flow on a link $q_{m}$ can be obtained from:

$$
q_{m}=u w /\left(s_{0}(u+w)\right)
$$

The proposed framework consists in four basic modules as depicted in figure 2. 


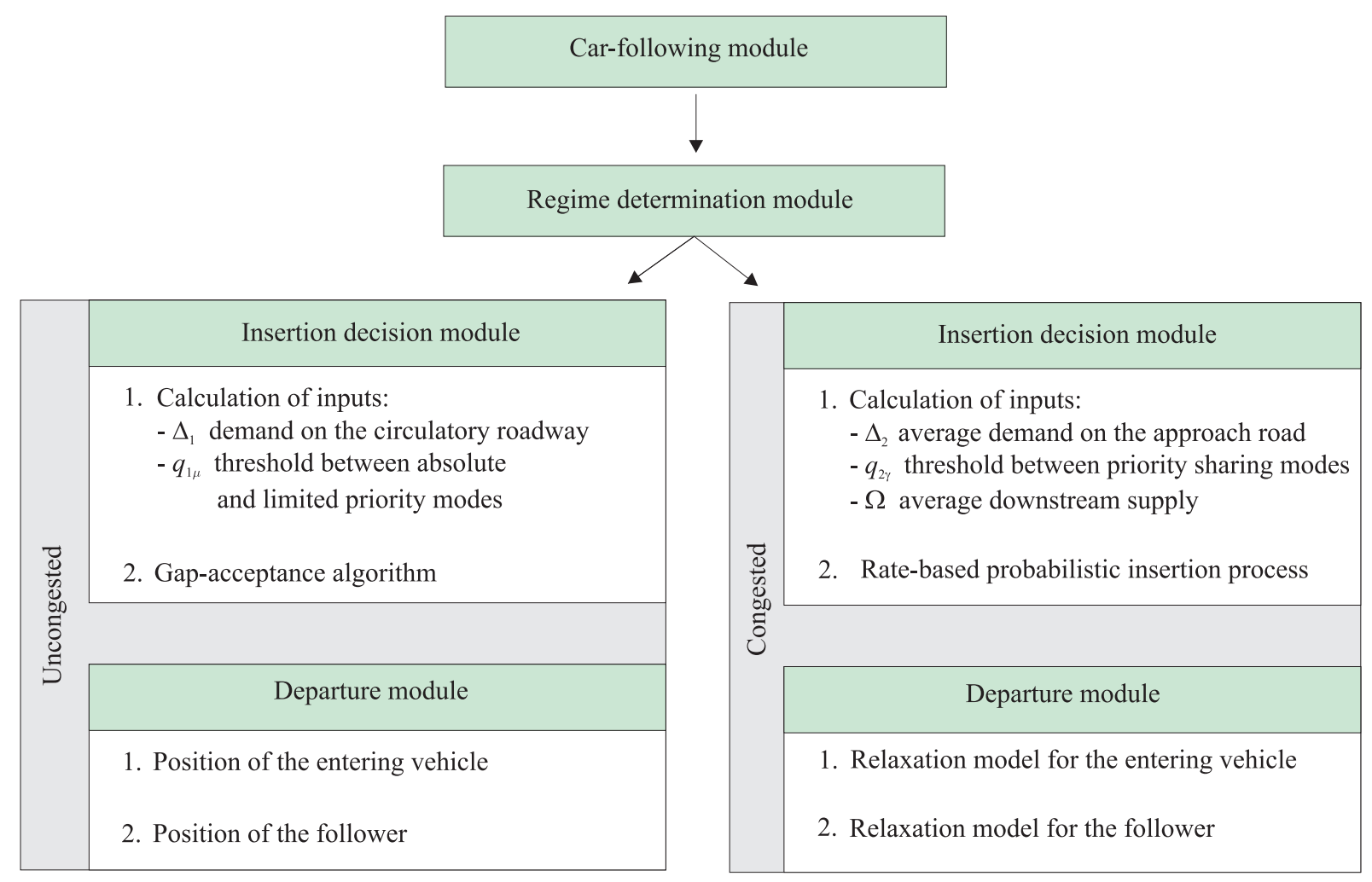

Fig.2. Flowchart of the algorithm

Positions of all vehicles except the follower and the entering vehicle are updated according to the car-following module. The regime determination module aims at assessing the traffic conditions on link $C_{i}$ to specify whether the gap-acceptance or the average priority sharing process occurs at the conflict point. The insertion decision module determines whether the entering vehicle could insert into the circulatory roadway during the simulation time-step, $\Delta t$. Finally, the departure module calculates the positions of the entering vehicle and the follower at the end of the simulation time-step. The next sections will detail these modules for each regime. 


\subsection{Car-following module}

\subsubsection{General principle}

The proposed microscopic framework can handle with any car-following model provided that: (i) it allows for non-equilibrium spacings between vehicles and (ii) it models a gradual increase of these spacings to desired values. This is usually defined as a relaxation procedure (Cohen, 2004; Hidas, 2005). In this study, this procedure is achieved though an extension of the Newell's simplified model (Newell, 2002). Its derivation is the result of studies leaded by Laval and Leclercq (2007) on the relaxation model and by Leclercq et al. (2007) on the Lagrangian formulation of the variational principle (Daganzo 2005). The position of vehicle $n$ at time $t+\Delta t, x_{n}^{t+\Delta t}$, is given as the minimum between the position it is willing to reach (freeflow term) and the position it cannot overpass due to the downstream vehicle $n-1$ (congested term):

$$
x_{n}^{t+\Delta t}=\left\{\begin{array}{l}
\min [\underbrace{x_{n}^{t}+\min \left(u, v_{n}^{t}+a \Delta t\right) \Delta t}_{\text {free-flow term }} ; \underbrace{x_{n-1}^{t}+v_{n-1}^{t+\Delta t} \Delta t-\Delta N_{n}^{t+\Delta t} s\left(v_{n-1}^{t+\Delta t}\right)}_{\text {congested term }}] \text { if } \Delta t \geq \frac{s_{0} \Delta N_{n}^{t}}{w} \\
\min [\underbrace{x_{n}^{t}+\min \left(u, v_{n}^{t}+a \Delta t\right) \Delta t}_{\text {free-flow term }} ; \underbrace{(1-\alpha) x_{n}^{t}+\alpha x_{n-1}^{t}+v_{n-1}^{t+\Delta t} \Delta t-\Delta N_{n}^{t+\Delta t} s\left(v_{n-1}^{t+\Delta t}\right)}_{\text {congested term }}] \text { otherwise }
\end{array}\right.
$$

In equation (2), $a$ is the maximum acceleration common to all vehicles; $v_{n}^{t+\Delta t}$ is the velocity of vehicle $n$ between $t$ and $t+\Delta t ; s(v)=s_{0}(w+v) / w$ is the equilibrium spacing corresponding to speed $v$ in congestion; $\Delta N_{n}^{t}$ is the ratio between the current spacing in front of vehicle $n$ and the equilibrium spacing associated with the speed of its leader; $\alpha=w \Delta t /\left(s_{0} \Delta N_{n}^{t}\right)$ is a nondimensional coefficient. 
If $\Delta N_{n}^{t}=1$, vehicle $n$ is in equilibrium and equation (2) is equivalent to the Newel's model. Yet, because of aggressive insertions, $\Delta N_{n}^{t}$ can become strictly inferior to 1 . In this case, the relaxation process of vehicle $n$ is introduced. It is characterized by a gradual increase in time of $\Delta N_{n}^{t}$ since the beginning of the relaxation process at time $t_{0}$ and location $x_{0}$ :

$$
\left\{\begin{array}{l}
\Delta N_{n}^{t+\Delta t}=\min \left[1, \Delta N_{n}^{t}+\min \left(\Delta N_{n}^{t}\left(v_{n-1}^{t+\Delta t}-v_{n-1}^{t}\right)+\mathcal{E}, v_{n-1}^{t+\Delta t}\right) \Delta t / s\left(v_{n-1}^{t+\Delta t}\right)\right] \\
\Delta N_{n}^{t_{0}}=\left(x_{n-1}^{t_{0}}-x_{0}\right) / s\left(v_{n-1}^{t+\Delta t}\right)
\end{array}\right.
$$

The parameter $\varepsilon$ corresponds to the difference in speed the non-equilibrium vehicle $n$ is willing to accept with respect to its leader in order to recover an equilibrium spacing. It was found in Leclercq et al. (2007b) that an average value of $\varepsilon$ can provide accurate vehicle trajectories.

For computational convenience, vehicle positions are set back to zero at the beginning of each new link. Moreover, to ensure stability of the numerical scheme (2), we assume that the Courant-Friedrich-Lewy's condition is met: $\Delta t \leq \frac{s_{0}}{w}$.

\subsection{2. $\quad$ Approximated velocity}

In equations (2) and (3), the term $v_{n-1}^{t+\Delta t}$ simplifies if $\Delta N_{n}^{t+\Delta t}=1$ and the Courant-FriedrichLewy's condition is satisfied as an equality. Indeed, in this case, $v_{n-1}^{t+\Delta t} \Delta t-\Delta N_{n}^{t+\Delta t} s\left(v_{n-1}^{t+\Delta t}\right)=s_{0}$. Otherwise, $v_{n-1}^{t+\Delta t}$ should be calculated from the position of vehicle $n-1$ at the end of the current time-step. Consequently, vehicle positions have to be updated in ascending order. This is troublesome given the looping nature of roundabouts. To overcome this issue, the velocity of 
vehicle $n-1$ during the current time-step should be approximated from already known positions.

The variational principle tells us that the position of vehicle $n-1$ after a time-period $\Delta T=s_{0} \Delta N_{n-1}^{t} / w$ is $x_{n-1}^{t+\Delta T}=x_{n-2}^{t}-s_{0} \Delta N_{n-1}^{t}$. If $\Delta T$ is higher than $\Delta t, v_{n-1}^{t+\Delta t}$ can be exactly estimated during the time-step. Otherwise, it is assumed that vehicle $n-1$ does not overpass $x_{n-1}^{t+\Delta T}$ at the end of the time-step. In this case, the approximated velocity is a lower bound of the effective one. Consequently, more stringent constraints are imposed on vehicle $n$ which ensures consistency of vehicle spacings at the end of the time-step. After re-arrangement of equation (2) we obtain:

$$
v_{n-1}^{t+\Delta t}=\left\{\begin{array}{l}
\min \left[u ; \frac{x_{n-2}^{t}-x_{n-1}^{t}}{\Delta t}-\frac{s_{0} \Delta N_{n-1}^{t}}{\Delta t}\right] \text { if } \Delta t \geq \frac{s_{0} \Delta N_{n-1}^{t}}{w} \\
\min \left[u ; \alpha \frac{x_{n-2}^{t}-x_{n-1}^{t}}{\Delta t}-w\right] \text { otherwise }
\end{array}\right.
$$

It should be stressed that the approximated velocity only differs from the effective one when $\Delta t \geq \frac{s_{0} \Delta N_{n-1}^{t}}{w}$ and vehicle $n-1$ is not in equilibrium.

By relaxing the car-following model dependence to current time-step speed values, positions of all vehicles can be updated in an arbitrary order. It is a convenient property for the roundabout model to be integrated into a traffic flow simulation package of a whole network. In the sequel, we will describe the next modules which allow for updating the positions of the follower and the entering vehicle.

\subsection{Regime determination module}

The goal of this module is to specify whether downstream traffic conditions are in uncongested or in congested regime. 
Step 1: check if there is a vehicle standing as the leader: if not the regime is uncongested and the algorithm stops

Step 2: check if there is a vehicle standing as the follower: if not the regime is uncongested and the algorithm stops

Step 3: compute the free-flow and the congested terms in equation (2) for the leader

Step 4: if the congested term is lower than the free-flow term, the regime is congested; otherwise it is uncongested

\subsection{Insertion decision module}

For each regime, the goal is to calculate the available time for insertion within the simulation time-step, $t_{a} \in[0, \Delta t]$. If $t_{a}>0$, the entering vehicle can insert into the circulating roadway and the insertion time $t_{0}$ is $t+\Delta t-t_{a}$. On the contrary, if $t_{a}=0$, the insertion is impossible during the time-step.

\subsubsection{Uncongested regime}

\section{a. Gap-acceptance algorithm}

The microscopic framework can deal with any gap-acceptance logic included into classical commercial software. We chose to work with an easy-to-calibrate algorithm to ensure that the simulated results are consistent with an already validated analytical model as recommended by Smith et al. (2007). The insertion takes place during the time-step if two criteria are met:

(i) the leader should have crossed the conflict point for a time equal to the follow-up time, $t_{f}$ 
(ii) the follower should be beyond a distance $d_{\text {lag }}$ of the conflict point.

The key principle of the insertion decision module is to compute $d_{\text {lag }}$ in terms of the average demand flow on the circulatory roadway, $\Delta_{1}$. This guarantees the consistency of the simulated circulating and entry flows, $q_{1}$ and $q_{2}$ with a well-established analytical model proposed by Troutbeck (2002). In this model, $q_{1}$ and $q_{2}$ are obtained by projecting the initial demand levels $\left(\Delta_{1}, \Delta_{2}\right)$ onto a capacity curve, $C$, as depicted in figure 3.

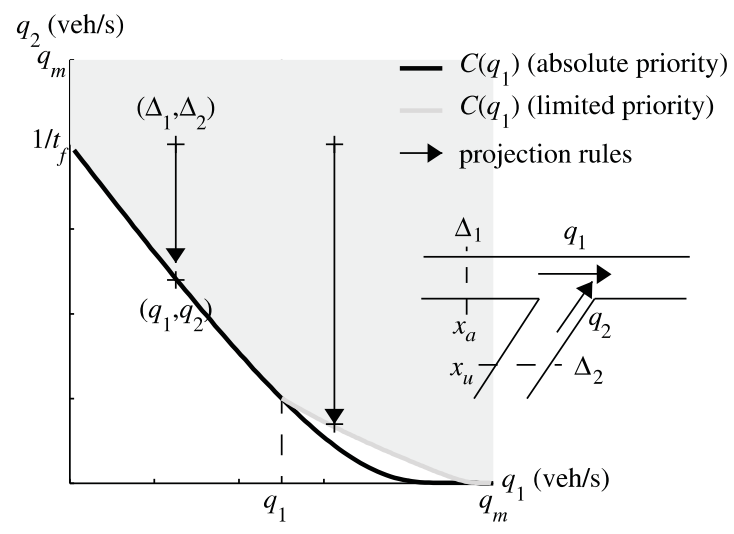

Fig.3. Capacity curve produced by the chosen gap-acceptance algorithm

An asset of the gap-acceptance algorithm is to reproduce the two priority modes observed by Troutbeck and Kako (1999) with respect to the entering vehicle's aggressiveness. If $\Delta_{1}$ is lower than a given threshold, $q_{1 \mu}$, the insertion of the entering vehicle do not affect the circulating flow (absolute priority mode). On the contrary, if $\Delta_{1}$ exceeds $q_{1 \mu}$, the entering vehicle adopts a more aggressive behaviour and can force the follower to slow down (limited priority mode). In our model, $q_{1 \mu}$ is calculated from $t_{f}, q_{m}^{C}$ and a parameter called the dynamic priority ratio, $\mu$ (for more details see Chevallier and Leclercq, 2007):

$$
q_{1 \mu}=\frac{1}{t_{f} \mu+1 / q_{m}^{C}}
$$


The insertion decision module consists in assessing conditions (i) and (ii) within the simulation time-step by interpolating vehicle positions as detailed below.

Step 1: calculate the entering vehicle's position at the end of the simulation time-step, $x_{e}^{t+\Delta t}$, according to the free-flow term in equation (2)

Step 2: if $x_{e}^{t+\Delta t}$ is upstream of the conflict point, the entering vehicle cannot reach the conflict point during the time-step. Hence $t_{a}=0$ and the algorithm stops. Otherwise, calculate the required time $t_{r} \in[0, \Delta t]$ for reaching the conflict point, from interpolation between $x_{e}^{t}$ and $x_{e}^{t+\Delta t}$ at free-flow speed

Step 3: calculate $\Delta_{1}(t)$ by computing the average of passing flows, $q_{u}$, by a downstream location, $x_{u}$, during the last $T$-seconds

$$
\Delta_{1}(t)=\min \left[q_{m}^{I} ; \operatorname{mean}_{s \in[t-T, t]}\left(q_{u}(s)\right)\right]
$$

Step 4: calculate $d_{\operatorname{lag}}(t)$ depending on $\Delta_{1}(t)$

$$
d_{\mathrm{lag}}(t)=\left\{\begin{array}{l}
u^{I} / q_{m}^{I} \quad \text { if } \Delta_{1}(t) \leq q_{1 \mu} \\
\frac{u^{I}}{q_{m}^{I}}\left(1-\frac{\Delta_{1}(t)-q_{1 \mu}}{q_{m}^{I}-q_{1 \mu}}\right) \text { otherwise }
\end{array}\right.
$$

Note that the first term represents the absolute priority mode and the second one the limited priority mode.

Step 5: calculate the follower's position in case of no insertion, $\hat{x}_{f}^{t+\Delta t}$, according to equation (2) with the leader $l$ standing as vehicle $n-1$ 
Step 6: calculate the remaining time $t_{s}$ for condition (i) to be satisfied. If $t_{s}>\Delta t$, the insertion cannot take place during the time-step and $t_{a}=0$. Otherwise, condition (ii) should be assessed at a time equal to the maximum between $t_{s}$ and $t_{r}$.

Step 7: calculate the follower's position, $x_{f}^{t+\max \left(t_{s}, t_{r}\right)}$, from interpolation between $x_{f}^{t}$ and $\hat{x}_{f}^{t+\Delta t}$ at constant speed. If $x_{f}^{t+\max \left(t_{s}, t_{r}\right)}$ falls within a distance $d_{\mathrm{lag}}(t)$ from the conflict point, the insertion is impossible and $t_{a}=0$. Otherwise, the entering vehicle can insert as soon as condition (i) is satisfied and it reaches the conflict point; thus: $t_{a}=\Delta t-\max \left(t_{s}, t_{r}\right)$.

\section{b. Including additional disturbance due to exiting vehicles}

Several studies (Hagring, 2001; Mereszczak et al., 2006) have highlighted the influence of vehicles leaving the roundabout towards link $D_{i}$ (exiting vehicles) on the entry capacity. Particularly, for a constant circulating flow on link $I_{i}$, the entry capacity is decreasing when the flow towards $D_{i}$ is increasing. Yet, the disturbance caused by exiting vehicles reveals to be of lower magnitude than the one caused by non-exiting vehicles (circulating vehicles). These phenomena are partly reproduced by the chosen gap-acceptance algorithm. Indeed, if $d_{\text {lag }}$ is higher than $L^{I}$, the follower impedes the insertion of the entering vehicle until the beginning of the time-step during which it crosses: (i) the boundary between $C_{i-1}$ and $D_{i}$ if it is an exiting vehicle; (ii) the conflict point if it is a circulating vehicle. However, in real traffic situations, if the follower leaves the circulatory roadway within $[t, t+\Delta t]$, it may still disturb the insertion process during a time-interval $[t, t+m \Delta t]$. The parameter $m$ depends on the time needed for the entering vehicle to detect the follower as an exiting vehicle. This time can vary amongst drivers. In our framework, this phenomena is reproduced by assuming an average 
probability $\beta$ for the entering vehicle to fail to detect the exit of a vehicle within the interval $[t, t+m \Delta t]$. The gap-acceptance algorithm is then completed by the following steps:

Step 1: check if any vehicle has left the roundabout toward link $D_{i}$ within the interval $[t-(m-1) \Delta t, t+\Delta t]$. If not, $t_{a}$ is not modified and the algorithm stops. Otherwise, go to step 2.

Step 2: specify if the entering vehicle has already detected the exit of the vehicle by generating a random number $z \in[0,1]$. If $z \leq \beta$, the exit has not yet been detected: the exiting vehicle still disturbs the insertion process and $t_{a}$ is set equal to 0 . Otherwise, $t_{a}$ is not modified.

For a simulation time-step of about $1 \mathrm{~s}, m$ can be fixed equal to 1.

\subsubsection{In congested regime}

In congested regime, circulating vehicles are queuing downstream and upstream of the conflict point. To allocate the downstream capacity, $\Omega$, between entry and circulating flows, Daganzo (1995) has proposed a distribution scheme based on the demand-supply theory. This model is fully consistent with observations made by Cassidy and Ahn (2005) and Troutbeck (2002). To compute the insertion rate $q_{2}$, it accounts for two kinds of behaviours depending on the traffic conditions on the approach link:

(i) in case of a queue, there is an average priority sharing process between both streams defined by the ratio $\gamma$;

(ii) in case of isolated arrivals with low average flow, approaching vehicles force their way into the circulatory roadway. 
These behaviours correspond to the two different projection rules of $\left(\Delta_{1}, \Delta_{2}\right)$ onto the capacity curve $C$ represented in figure 4 :

(i) $\left(q_{1}, q_{2}\right)=\left(q_{1 \gamma}, q_{2 \gamma}\right)$;

(ii) $\left(q_{1}, q_{2}\right)=\left(C^{-1}\left(\Delta_{2}\right), \Delta_{2}\right)$.

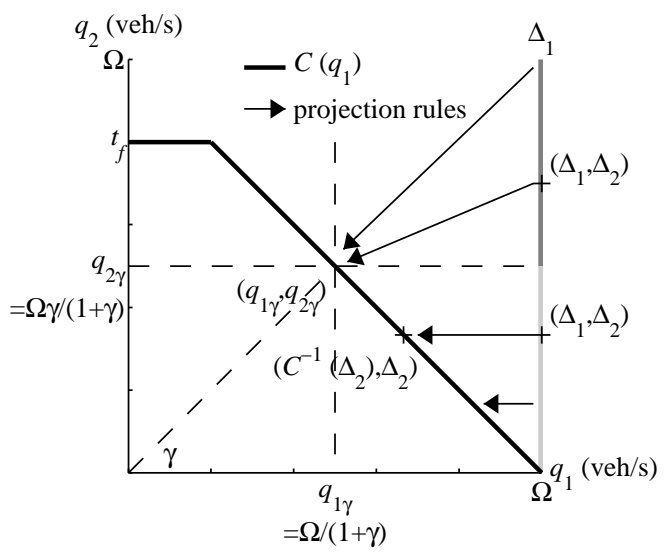

Fig.4. Insertion rate $q_{2}$ obtained by the Daganzo's model

As discussed in the introduction part, classical gap-acceptance algorithms fail to reproduce these behaviours. Up to now, the only available models for capturing these phenomena are multi-stage gap-acceptance models (Ahmed, 1999; Hidas, 2005; Choudhury et al., 2007). They explicitly consider the anticipatory aspect of cooperation or aggressiveness amongst drivers through several binary choice processes. For this, they require both estimates of future traffic state variables and storage of previous driver's decisions.

In this study, we aim at developing a simpler insertion decision model for reproducing the Daganzo's model. It consists in describing the insertion decision outcome as the result of a random experiment, in the spirit of the lane-changing model proposed by Laval and Leclercq (2008). For this, one needs to calculate the probability for an entering vehicle to insert during the time-step, $p^{t+\Delta t}$. It is obtained from $q_{2}$ and thus depends on the merging type behaviour (Chevallier and Leclercq, 2008): 


$$
p^{t+\Delta t}=q_{2}(t) \Delta t
$$

Then, the discrete outcome of the insertion process is specified according to a Bernoulli experiment of probability of success $p^{t+\Delta t}$. This ensures the consistency between the simulated average insertion rate on a given period and $q_{2}$. The following steps synthesise the insertion decision module.

Step 1: determine traffic conditions on the approach link by using the same approach as in the regime determination module. Particularly, if the free-flow term of the entering vehicle's position is lower than the congested term, traffic is uncongested; otherwise it is congested.

Step 2: calculate the average demand on the approach leg, $\Delta_{2}(t)$ by computing the average of passing flows, $q_{a}$, by a downstream location, $x_{a}$, during the last $T$-seconds

$$
\Delta_{2}(t)=\min \left[q_{m}^{A} ; \operatorname{mean}_{s \in[t-T, t]}\left(q_{a}(s)\right)\right]
$$

Note that if traffic on the approach link is congested $\Delta_{2}(t)$ is set equal to $q_{m}^{A}$.

Step 3: calculate the downstream capacity, $\Omega(t)$, from the average of passing flows, $q_{d}$, by a downstream location, $x_{d}$, during the last $T$-seconds:

$$
\Omega(t)=\min \left[q_{m}^{C} ; \operatorname{mean}_{s \in[t-T, t]}\left(q_{d}(s)\right)\right]
$$

Step 4: calculate the boundary, $q_{2 \gamma}(t)$ between both merging-type behaviours of the Daganzo's model (see figure 4)

$$
q_{2 \gamma}(t)=\frac{\Omega(t) \gamma}{1+\gamma}
$$


Step 5: specify the insertion rate $q_{2}(t)$ depending on the merging-type behaviour (see figure 4)

$$
q_{2}(t)=\left\{\begin{array}{l}
\min \left[1 / t_{f}, q_{2 \gamma}(t)\right] \text { if } \Delta_{2} \geq q_{2 \gamma} \\
\Delta_{2}(t) \text { otherwise }
\end{array}\right.
$$

Step 6: calculate $p^{t+\Delta t}$ from equation (6)

$$
p^{t+\Delta t}=\left\{\begin{array}{l}
\min \left[1 / t_{f} ; q_{2 \gamma}\right] \Delta t \text { if } \Delta_{2} \geq q_{2 \gamma} \\
1 \text { otherwise }
\end{array}\right.
$$

Step 7: calculate the discrete outcome of the insertion process by generating a random number $z \in[0,1]$. If $z \leq p^{t+\Delta t}$, the insertion takes place and $t_{a}=\Delta t$. Otherwise, the entering vehicle should wait and $t_{a}=0$.

\subsection{Departure module}

As soon as the available time for insertion during the time-step is specified, positions of the follower and the entering vehicle can be updated. In uncongested regime, like in any classical microscopic model, the car-following algorithm imposes constraints on the insertion decision module to avoid inconsistent vehicle trajectories. On the contrary, in congested regime, direct links between the car-following algorithm and the insertion decision model should be released for ensuring consistency between the simulated average insertion flow and the Daganzo's model.

\subsubsection{In uncongested regime}

To allow for an insertion during the time-step when $t_{a}>0$, two conditions induced by the gap-acceptance algorithm should be met: 
(i) on the entering vehicle: its position imposed by the leader at the end of the insertion manoeuvre should not be upstream of the conflict point;

(ii) on the follower: the insertion of the entering vehicle should not cause the follower to drive backwards.

In uncongested regime, both the follower and entering vehicle are in equilibrium. Thus their positions are always updated according to the second case in equation (2) with $\Delta N_{n}^{t+\Delta t}=1$.

Step 1: calculate the distance travelled by vehicle $e$ onto the circulating roadway from the insertion time $t_{0}$ to the end of the time-step corresponding to $t_{0}+t_{a}$. For this, use equation (2) with $\Delta t=t_{a}$ and $t=t_{0}$. Note that $x_{e}^{t_{0}}=0$.

$$
x_{e}^{t+\Delta t}=\min \left[\min \left[u^{C} ; v_{e}^{t_{0}}+a t_{a}\right] t_{a} ; \alpha x_{l}^{t_{0}}+v_{l}^{t+\Delta t} t_{a}-s\left(v_{l}^{t+\Delta t}\right)\right]
$$

with $\left\{\begin{array}{l}v_{e}^{t_{0}}=\frac{L^{A}-x_{e}^{t}}{t_{0}} \\ v_{l}^{t+\Delta t}=\frac{x_{l}^{t+\Delta t}-x_{l}^{t}}{\Delta t} \\ x_{l}^{t_{0}}=x_{l}^{t}+t_{0} v_{l}^{t+\Delta t}\end{array}\right.$

It should be noticed that $v_{l}^{t+\Delta t}$ can be exactly computed since the position of the leader $l$ at time $t+\Delta t$ is known from the car-following module.

Step 2: if $x_{e}^{t+\Delta t}<0$, condition (i) is not satisfied and vehicle $e$ should wait: $x_{e}^{t+\Delta t}=L^{A}$. The follower can advance up to the position calculated in case of no insertion, $\hat{x}_{f}^{t+\Delta t}$ (see section 2.4.1.a.). Otherwise, go to step 3. 
Step 3: The position of the follower should be calculated to account for its new leader $e$. For this, we set $\Delta t=t_{a}$ and $t=t_{0}$ in equation (2). Note that $x_{e}^{t_{0}}=L^{P}$.

$$
x_{f}^{t+\Delta t}=\min \left[x_{f}^{t}+\min \left[u^{P}, v_{f}^{t}+a t_{a}\right] t_{a} ;(1-\alpha) x_{f}^{t_{0}}+\alpha x_{e}^{t_{0}}+v_{e}^{t+\Delta t} t_{a}-s\left(v_{e}^{t+\Delta t}\right)\right]
$$

with $\left\{\begin{array}{l}x_{f}^{t_{0}}=x_{f}^{t}+t_{0} \frac{\hat{x}_{f}^{t+\Delta t}-x_{f}^{t}}{\Delta t} \\ v_{e}^{t+\Delta t}=\frac{x_{e}^{t+\Delta t}}{t_{a}}\end{array}\right.$

Step 4: if $x_{f}^{t+\Delta t}<x_{f}^{t}$ condition (ii) is not satisfied and the insertion cannot take place (see step 2 for final positions of vehicles $e$ and $f$ ). Otherwise, go to step 5.

Step 5: as conditions (i) and (ii) are met, the insertion can occur: the follower and the entering vehicle advance up to their calculated positions at steps 1 and 3.

\subsubsection{In congested regime}

In congested regime, if the time-step is available, the entering vehicle insert into the circulating roadway at the beginning of the time-step $\left(t_{0}=t\right)$. Both the follower and the entering vehicle may switch to a non-equlibrium state at $t_{0}$. Hence, the departure model consists in two steps for updating the positions of either $e$ or $f$. First, the ratio $\Delta N_{e}^{t}$ (respectively $\Delta N_{f}^{t}$ ) representing how close to the equilibrium spacing is the effective spacing in front of $e$ (respectiveley $f$ ) should be computed with vehicle positions at the insertion time. Then, this ratio should be calculated at the end of the time-step from equation (3) before updating the vehicle's position from equation (2).

Step 1: compute $\Delta N_{e}^{t}$ 


$$
\Delta N_{e}^{t}=x_{l}^{t_{0}} / s\left(v_{l}^{t+\Delta t}\right)
$$

Step 2: compute $\Delta N_{e}^{t+\Delta t}$ from equation (3) with $n=e, n-1=l$ and $v_{n-1}^{t+\Delta t}=v_{l}^{t+\Delta t}$

Step 3: update the entering vehicle's position, $x_{e}^{t+\Delta t}$, according to equation (2)

Step 4: compute $\Delta N_{f}^{t}$

$$
\Delta N_{f}^{t}=x_{f}^{t_{0}} / s\left(v_{e}^{t+\Delta t}\right)
$$

Step 5: compute $\Delta N_{f}^{t+\Delta t}$ from equation (3) with $n=f, n-1=e$ and $v_{n-1}^{t+\Delta t}=x_{e}^{t+\Delta t} / \Delta t$

Step 6: update the follower's position, $x_{f}^{t+\Delta t}$, according to equation (2) with $x_{n-1}^{t}+v_{n-1}^{t+\Delta t} \Delta t=x_{e}^{t+\Delta t}+L^{P}$

\subsection{Synthesis of the model parameters}

An asset of the proposed framework is to be parsimonious. Indeed, most of the parameters used in the previous sections are invariant in an urban context.

In the car-following module, the minimum spacing in congestion $s_{0}$ only depends on the number of lanes. The reference value per lane is usually comprised between $4.5 \mathrm{~m}$ and $5.5 \mathrm{~m}$ (Leclercq, 2005). The value of $w$ can be deduced from equation (1) by assuming that the maximum flow on urban link is equal to $0.5 \mathrm{veh} / \mathrm{s}$ per lane. It was also shown in Leclercq et al. (2007b) that the relaxation process can be accurately reproduced with a value of $\varepsilon$ equal to $0.55 \mathrm{~m} / \mathrm{s}$. Finally, to model bounded acceleration, the maximum acceleration $a$ can be chosen in a range of $1 \mathrm{~m} / \mathrm{s}^{2}$ to $2.5 \mathrm{~m} / \mathrm{s}^{2}$ as commonly found in the literature. Consequently, the only car-following parameter needed to be calibrated from on-field observations is the free-flow speed on each link. 
Similarly, in the insertion decision module, several parameters do not need to be modified. For instance, the distances $x_{u}, x_{d}$ and $x_{a}$ at which the demand flows and the downstream capacity are calculated do not influence the simulated results. We chose a default value of $10 \mathrm{~m}$ from the conflict point. The aggregation period $T$ for computing these flows is also of little impact provided that it is short enough to account for dynamics. It is taken equal to $30 \mathrm{~s}$ by default. Thus, the only insertion decision parameters needed to be adapted to on-field conditions are:

- the probability $\beta$ for an exiting vehicle to impede the insertion process during the current time-step which essentially depends on geometric characteristics;

- the follow-up time $t_{f}$ which represents the average time-interval between two insertions when there is no vehicle on the circulating roadway;

- the dynamic priority ratio $\mu$ deduced from equation (5) by observing the average circulating flow $q_{1 \mu}$ beyond which aggressive insertion manoeuvres are observed;

- the priority ratio in congestion $\gamma$ obtained by assessing the average advancing flows from the circulatory roadway and the approach leg when traffic is congested.

\section{VALIDATION}

In this section, the roundabout model is applied to on-field situations in France. Data were first collected on a whole roundabout (Bellefontaine) in Toulouse in order to validate the overall algorithm. However, flows on the approach leg and the circulatory roadway are usually too low for a complete assessment of the model's merging ability. Hence, the validation process was completed by two other data collection studies. They were performed on only one merging area of two near-to-capacity roundabouts (Solaize and Villeurbanne) in 
Lyon. They allow for checking the consistency between the observed average delays on the approach link and the simulation results.

\subsection{Data collection process}

Two methods were used to collect vehicle identities and passing times at different positions of the roundabout or the merging areas:

(i) the video recording of vehicle movements combined with an image processing software;

(ii) the use of pocket laptop computers by several observers.

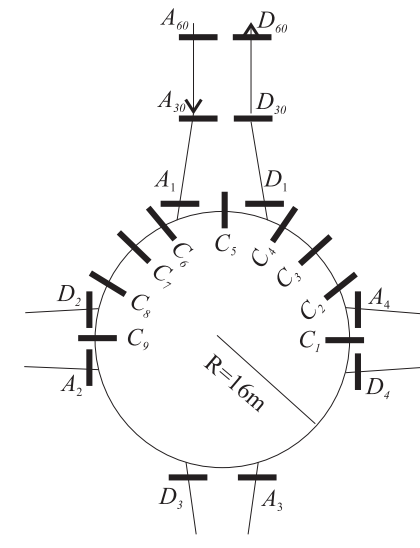

a: Bellefontaine roundabout

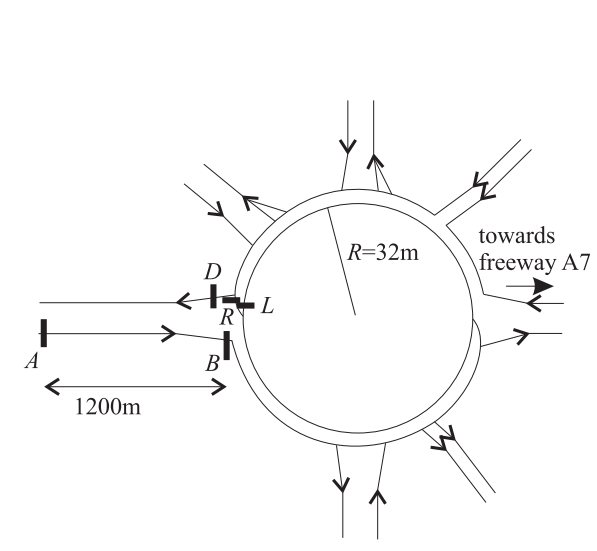

b: Solaize merging area

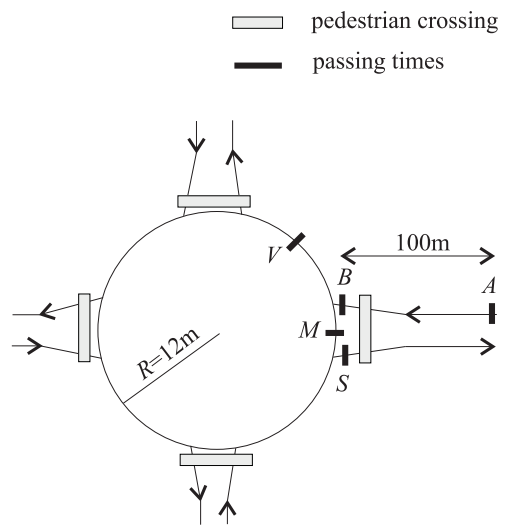

c: Villeurbanne merging area

Fig.5. Data collection process

Method (i) was used for the Bellefontaine roundabout; method (ii) for the Villeurbanne merging area and a combination of both for the Solaize merging area. Figure 5 shows the location of the data collection points.

\subsection{Parameter calibration}

Table 1 summarizes the parameter values fitted for each studied site. Note that the insertion module parameters fall in the range of values commonly recommended in the literature 
(HCM, 2006). For each simulation run, creation times of vehicles on approach links exactly correspond to passing times recorded at type- $A$ collection points. Moreover, all vehicles are assigned with a departure link thanks to the track of vehicle identities.

\begin{tabular}{|c|c|c|c|c|c|c|}
\hline & & units & Bel. & $\begin{array}{c}\text { Sol. } \\
\text { (cong.) }\end{array}$ & $\begin{array}{c}\text { Sol. } \\
\text { (uncong.) }\end{array}$ & Vil. \\
\hline \multirow{7}{*}{ 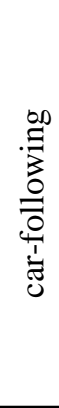 } & $s_{0} /$ lane & {$[\mathrm{m}]$} & 4.8 & 5.6 & 5.6 & 5.6 \\
\hline & $\varepsilon$ & {$[\mathrm{m} / \mathrm{s}]$} & 0.6 & 0.55 & 0.55 & 0.6 \\
\hline & $a$ & {$[\mathrm{~m} / \mathrm{s} 2]$} & 2.3 & 2.3 & 2.3 & 2.3 \\
\hline & $w^{A}=w^{D}$ & \multirow{2}{*}[\mathrm{m}/\mathrm{s}]{} & 3.2 & 3.26 & 3.26 & 3.5 \\
\hline & $w^{\mathrm{C}}=w^{I}$ & & 4.3 & 3.84 & 3.84 & 4.5 \\
\hline & $u^{A}=u^{D}$ & \multirow{2}{*}[\mathrm{m}/\mathrm{s}]{} & 12 & 18.8 & 18.8 & 14 \\
\hline & $u^{C}=u^{I}$ & & 5.3 & 10 & 10 & 7 \\
\hline \multirow{6}{*}{ 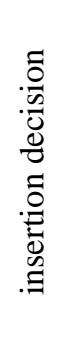 } & $x_{u}=x_{a}=x_{d}$ & {$[\mathrm{~m}]$} & 10 & 10 & 10 & 10 \\
\hline & $T$ & {$[\mathrm{~s}]$} & 30 & 30 & 30 & 30 \\
\hline & $\beta$ & $\%$ & 0 & 0.6 & 0.6 & 0.6 \\
\hline & $t_{f}$ & [s] & 3 & 2 & 2.45 & 2.2 \\
\hline & $\mu$ & no & 0.3 & 1 & 0.82 & 0.5 \\
\hline & $\gamma$ & no & 1 & 1 & 1 & 1 \\
\hline
\end{tabular}

Tab.1. Parameters values

\subsection{Overall model validation}

In order to validate the overall roundabout model, we used the data collected on the Bellefontaine roundabout. We have calculated the observed and simulated cumulative vehicle counts, $N(x, t)$, past any points $x \in\left\{A_{k}, C_{k}, D_{k}\right\}$ (see figure 5) by time $t$. Figure 6 depicts eight of these $\mathrm{N}$-curves in an oblique coordinate system to magnify their vertical displacements expressed in vehicles. The discrepancy between curves never exceeds 3 vehicles $(0.1 \%$ of the total entering vehicles). These results show evidence of the good general behaviour of the roundabout model. 

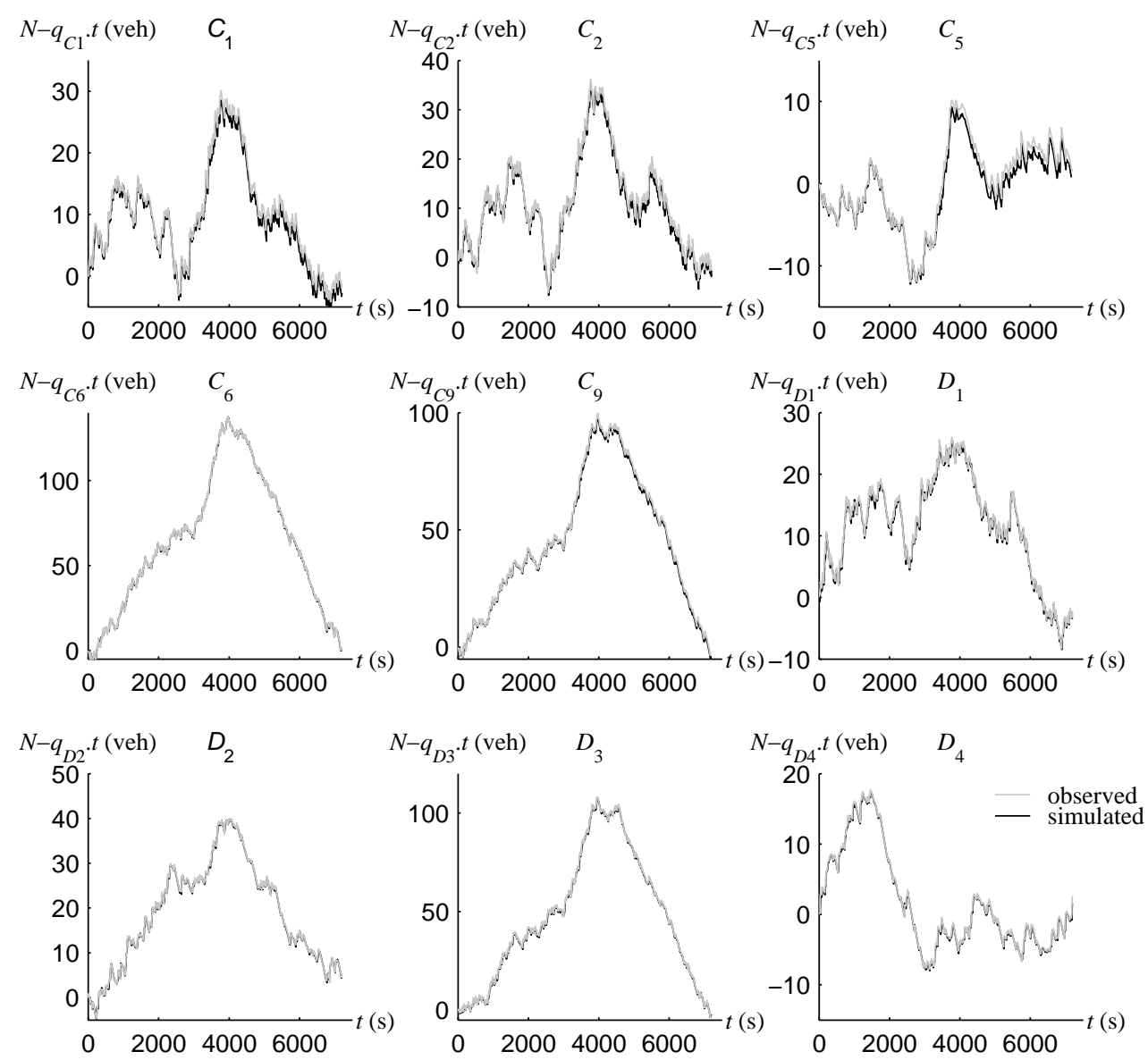

Fig.6. Validation of the overall model

\subsection{Merging algorithm validation}

To study in more details the merging ability of the roundabout model, we used experimental data collected on: (i) the Solaize merging area during a peak period for which the regime is merely congested; (ii) the Solaize merging area during an off-peak period for which the regime is merely uncongested; (iii) the Villeurbanne merging area during an entire two-hour period mixing both uncongested and congested regimes.

The following figure depict, for each case, the observed and simulated $N$-curves at location $B$ (see figure 5) in an oblique coordinate system. They also compare the observed and simulated average vehicle delays on the approach link for an aggregation period of respectively $5 \mathrm{~min}$ and $15 \mathrm{~min}$. 

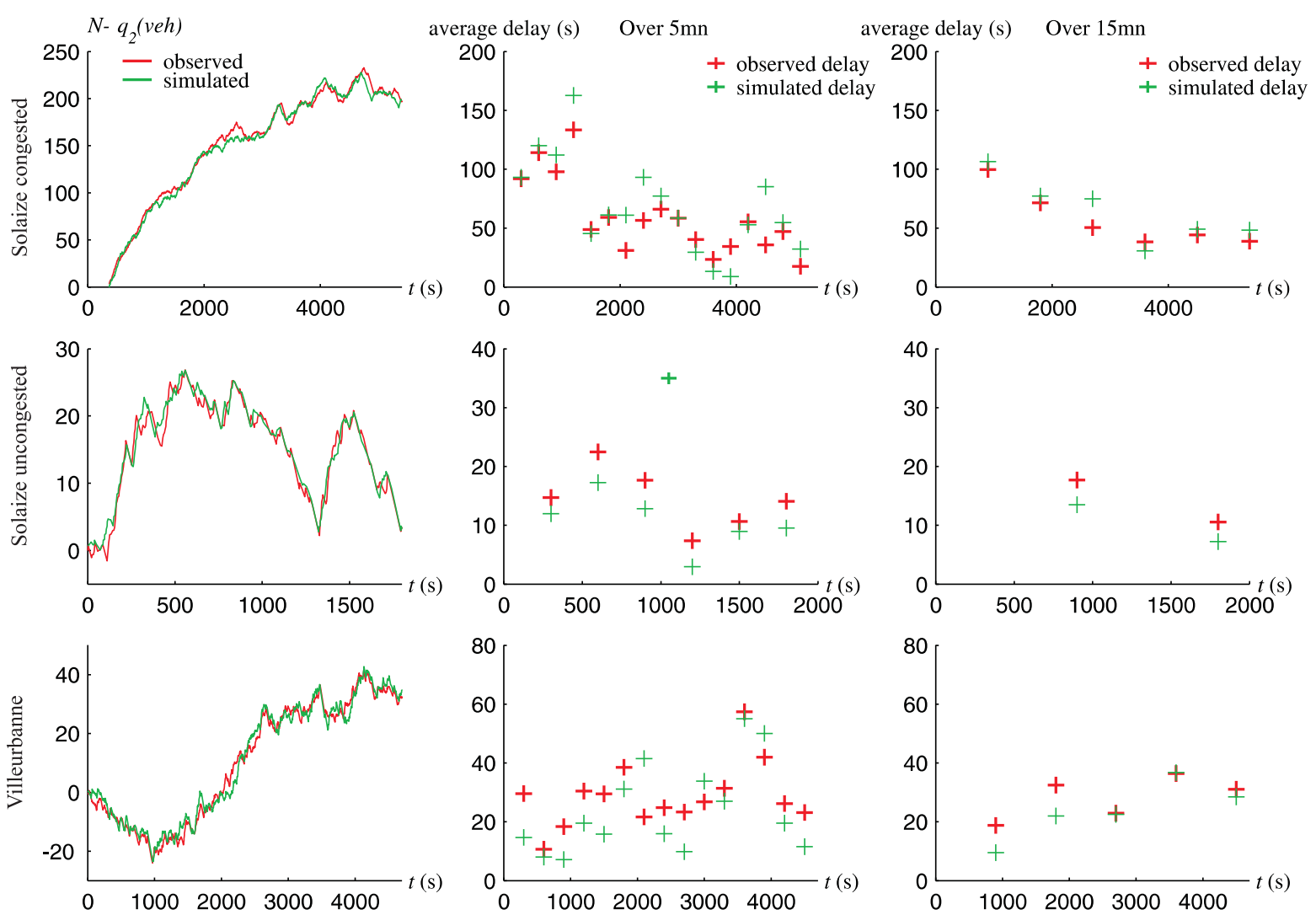

Fig.7. $N$-curves and delays at each site

\subsection{1. $\quad$ Solaize merging area during peak}

During the 1h30min-collection period (8:00am-9:30am), the maximum displacement between the simulated and the observed $N$-curves is about 15 veh over 1440 observed. This good match in entry flows is also reflected in figure 7 by consistent simulated average delays compared to experimental data. Particularly, for a $15 \mathrm{mn}$ aggregation period, model estimates usually falls within a $20 \%$ error interval around observed values. It should be noted that classical gapacceptance algorithm would completely fail to reproduce these delays since the circulatory roadway is merely always congested. 


\subsection{2. $\quad$ Solaize merging area during off-peak}

During the 30min-collection period (7:30am-8:00am), the simulated entering flows correctly fit the experimental data. Particularly, the discrepancy between observed and simulated $\mathrm{N}$ curves never exceeds 5 vehicles over 260 observed as presented in figure 8 . Simulated average delays are closed to the observed ones even if they are usually underestimated of about $4 \mathrm{~s}$.

\subsubsection{Villeurbanne merging area}

During the 1h30min-collection period (7:30am-9:00am), the simulated and observed $N$-curves never differ from more than 7 vehicles over 797 observed. The average delays also match quite accurately the experimental data as shown in figure 9, especially for a $15 \mathrm{~min}$ aggregation period.

\section{CONCLUSION}

Currently, most of microsimulations used to model roundabouts encompass gap-acceptance logics which are irrelevant to represent merging behaviours when a congestion spills back on the circulatory roadway. To overcome this issue, a new microscopic framework for singlelane roundabouts was proposed in this article. It can be easily integrated into a simulation package of a whole network since the car-following model does not require any predefined order for updating vehicle positions. It includes: (i) a module which specifies if the regime is congested or not; (ii) a classical gap-acceptance logic for representing merging behaviour in uncongested regime; (iii) a new insertion decision algorithm specifically developed to model merging behaviours in congested regime.The framework was validated in terms of flows and average delays for entering vehicles at different sites and for both peak and off-peak periods. 
To pursue the validation of the model, it could be worth studying the model performance in terms of other traffic performance indicators such as the geometric delay, the back of the queue location, the probability to stop or some percentile of the queue length distribution. Further research should also be conducted to extend this model to multi-lane roundabouts or to other types of unsignalized intersections.

\section{References}

Ahmed, K.I., 1999. Modeling driver's acceleration and lane changing behaviour. PhD dissertation, Department of Civil and Environmental Engineering, MIT.

Akçelik, R., 2005. Roundabout model calibration issues and a case study. Proceedings of Transportation Research Board National Roundabout Conference, Vail, Colorado.

Akçelik, R., Besley, M., 2001. Microsimulation and analytical methods for modelling urban traffic. Proceedings of the Conference on Advance Modeling Techniques and Quality of Service in Highway Capacity Analysis, Truckee, California.

Bared, J.G., Edara, P. K., 2005. Simulated capacity of roundabouts and impact of roundabout within a progressed signalized road. Proceedings of Transportation Research Board National Roundabout Conference, Vail, Colorado.

Cassidy, M.J., Ahn, S., 2005. Driver turn-taking behaviour in congested freeway merges. Transportation Research Record, 1934, 140-147.

Chevallier, E., Leclercq, L., 2008. Macroscopic investigation of microscopic merging models at unsignalized intersections. Proceedings of the Transportation Research Board, Washington, DC.

Chevallier,E., Leclercq, L., 2007. A macroscopic theory for unsignalized intersections, Transportation Research Part B 41 (10), 1139-1150.

Choudhury, C., Ben-Akiva, M.E., Toledo, T., Rao, A., Lee, G., 2007. State dependence in lane changing models. Proceedings of the 17th International Symposium on Transport Simulation, Ed.: Allsop, R.E., Bell, M.G.H., Heydecker, B.G., Elsevier, London, 711-733.

Cohen, S.L., 2004. Application of a relaxation procedure for lane changing in microscopic simulation models. Transportation Research Record, 1883, 50-58.

Daganzo, C., 1995. The cell transmission model, Part II: network traffic. Transportation Research Part B 29 (2), 79-93.

Daganzo, C.F., 2005. A variational formulation of kinematic waves: basic theory and complex boundary conditions. Transportation Research Part B, 39 (2), 187-196.

Hagring, O., 2001. Derivation of capacity equation for roundabout entry with mixed circulating and exiting flows. Transportation Research Record, 1776, 91-99.

Highway Capacity Manual, 2006. Proposed Draft Chapter 17, Part C: Roundabouts. Proceedings of the AHB40 committee, Transportation Research Board, Washington, DC. 
Hidas, P., 2005. Modelling vehicle interactions in microscopic simulation of merging and weaving. Transportation Research Part C 13 (1), 37-62.

Hidas, P., 2005b. Lane changing and merging under congested conditions in traffic simulation models. Urban Transport XI - Urban Transport and the Environment in the 21st Century, Ed.: Brebbia, C.A., Wadhwa, L.C., WIT Press, Southampton, Boston, 779-788.

Laval, J.A., Leclercq, L., 2007. Microscopic modeling of the relaxation phenomenon using a macroscopic lane-changing model. Transportation Research Part B, doi:10.1016/j.trb.2007.10.004

Leclercq, L., Laval, J., Chevallier, E., 2007. The Lagrangian coordinates and what it means for first order traffic flow models. Proceedings of the 17th International Symposium on Transport Simulation, Ed.: Allsop, R.E., Bell, M.G.H., Heydecker, B.G., Elsevier, London, 735-753.

Leclercq, L., Chiabaut, N., Laval, J., Buisson, C., 2007b. Relaxation phenomenon after changing lanes: an experimental validation with the NGSIM dataset. Transportation Research Record, 1999, 79-85.

Leclercq, L., 2005. Calibration of flow-density relationships on urban streets. Transportation Research Record, 1934, 226-234.

Mereszczak, Y., Dixon, M., Kyte, M., Rodegerdts, L., Blogg, M., 2006. Including exiting vehicles in capacity estimation at single-lane U.S. roundabouts. Proceedings of the Transportation Research Board, Washington, DC.

Robinson, B.W., 2000. Roundabout: an informational guide, Chapter 8: System consideration. Publication No. FHWA-RD-00-067.

Newell, G.F., 2002. A simplified car-following theory: a lower-order model. Transportation Research Part B, 36 (3), 195-205.

Smith, M. C., Sadek, A. W., Johnson, I., Aultman-Hall, L., Garder, P., 2007. Assessing the accuracy of roundabouts analysis procedures against field observations: preliminary results from a case study in Northern New England. Proceedings of the Transportation Research Board, Washington, DC.

Stanek, D., Milam, R. T., 2005. High-capacity roundabout intersection analysis: going around in circle. Proceedings of Transportation Research Board National Roundabout Conference, Vail, Colorado.

Tian, Z. Z., Urbanik II, T., Engelbrecht, R., Balke, K., 2003. Variations in capacity and delay estimated from microscopic traffic simulation models. Transportation Research Record, 1802, 23-31.

Troutbeck, R.J., 2002. The performance of uncontrolled merges using a limited priority process. Proceedings of the 15th International Symposium on Transportation and Traffic Theory, Ed.: Taylor, M., Pergamon, Amsterdam, 463-482.

Troutbeck, R.J., Kako, S., 1999. Limited priority merge at unsignalized intersections. Transportation Research Part A 33 (3-4), 291-304.

Trueblood, M. T., Dale, J., 2003. Simulating roundabouts with VISSIM. Proceedings of the 2nd Urban Street Symposium, Anaheim, California.

NYSDOT, 2002. Rodel 1: Interactive Roundabout Design. Joint copywright Rodel Software Ltd and Staffordshire County Council. 